PROCEEDINGS OF THE

AMERICAN MATHEMATICAL SOCIETY

Volume 142, Number 8, August 2014, Pages 2671-2681

S 0002-9939(2014)11972-1

Article electronically published on May 9, 2014

\title{
ASYMPTOTICS OF ORBITS OF A KOLMOGOROV TYPE PLANAR VECTOR FIELD WITH A FIXED NEWTON POLYGON
}

\author{
F. BEREZOVSKAYA \\ (Communicated by Sergei K. Suslov) \\ Dedicated to the memory of Professor A. M. Molchanov
}

\begin{abstract}
Using the Newton polygon technique we show that the orbits of a Kolmogorov type planar vector field, consisting of a finite sum of power terms, have power asymptotics while tending to the equilibria on the axes and on the boundary of the Poincaré sphere.
\end{abstract}

\section{INTRODUCTION}

Let us consider a two-dimensional vector field $\Omega$ defined on the Poincaré sphere [1] by a Kolmogorov type system

$$
\begin{aligned}
\Omega\left(V_{1}, V_{2}\right) \equiv \Omega(\nu): \frac{d \nu_{1}}{d t} & =V_{1}\left(\nu_{1}, \nu_{2}\right) \equiv \nu_{1} P_{1}\left(\nu_{1}, \nu_{2}\right), \\
\frac{d \nu_{2}}{d t} & =V_{2}\left(\nu_{1}, \nu_{2}\right) \equiv \nu_{2} P_{2}\left(\nu_{1}, \nu_{2}\right) .
\end{aligned}
$$

Here $\left(\nu_{1}, \nu_{2}\right) \equiv\left(x_{1}, x_{2}\right), V_{1}\left(\nu_{1}, \nu_{2}\right) \equiv X_{1}\left(x_{1}, x_{2}\right), V_{2}\left(\nu_{1}, \nu_{2}\right) \equiv X_{2}\left(x_{1}, x_{2}\right)$ for finite $x$, and power functions

$$
P_{1}\left(x_{1}, x_{2}\right)=\sum_{\mu, \nu} p_{\mu \nu} x_{1}^{\mu} x_{2}^{\nu}, \quad P_{2}\left(x_{1}, x_{2}\right)=\sum_{\mu, \nu} q_{\mu \nu} x_{1}^{\mu} x_{2}^{\nu}, \quad \mu \geq 0, \nu \geq 0, p_{\mu \nu}, q_{\mu \nu} \in \mathbf{R},
$$

consist of a finite number of summands with distinct powers $(\mu, \nu) \in \mathbf{R}_{+}^{2}$ :

$$
\begin{gathered}
\left(\nu_{1}, \nu_{2}\right) \equiv\left(z_{1}=1 / x_{1}, z_{2}=x_{2} / x_{1}\right) \\
V_{1}\left(\nu_{1}, \nu_{2}\right) \equiv Z_{1}\left(z_{1}, z_{2}\right)=z_{1} Q_{1}\left(z_{1}, z_{2}\right), \quad V_{2}\left(\nu_{1}, \nu_{2}\right) \equiv Z_{2}\left(z_{1}, z_{2}\right)=z_{2} Q_{2}\left(z_{1}, z_{2}\right), \\
Q_{1}\left(z_{1}, z_{2}\right)=-P_{1}\left(1 / z_{1}, z_{2} / z_{1}\right), \quad Q_{2}\left(z_{1}, z_{2}\right)=P_{2}\left(1 / z_{1}, z_{2} / z_{1}\right)-P_{1}\left(1 / z_{1}, z_{2} / z_{1}\right) \\
\left(\nu_{1}, \nu_{2}\right) \equiv\left(u_{1}=x_{1} / x_{2}, u_{2}=1 / x_{2}\right), \\
V_{1}\left(\nu_{1}, \nu_{2}\right) \equiv U_{1}\left(u_{1}, u_{2}\right)=u_{1} R_{1}\left(u_{1}, u_{2}\right), V_{2}\left(\nu_{1}, \nu_{2}\right) \equiv R_{2}\left(u_{1}, u_{2}\right)=u_{2} R_{2}\left(u_{1}, u_{2}\right), \\
R_{1}\left(u_{1}, u_{2}\right)=P_{1}\left(u_{1} / u_{2}, 1 / u_{2}\right)-P_{2}\left(u_{1} / u_{2}, 1 / u_{2}\right), R_{2}\left(u_{1}, u_{2}\right)=-P_{2}\left(u_{1} / u_{2}, 1 / u_{2}\right),
\end{gathered}
$$

respectively, close to equators of the Poincaré sphere $z_{1} u_{2}=0$.

Received by the editors June 21, 2012 and, in revised form, August 5, 2012.

2010 Mathematics Subject Classification. Primary 34E05.

Key words and phrases. Planar vector field, Poincaré sphere, orbit asymptotics, Newton polygon.

(C)2014 American Mathematical Society Reverts to public domain 28 years from publication 2671 
The vector field $\Omega(x)$ has a singular point $\left(x_{1}=0, x_{2}=0\right)$. This point is isolated only if $P_{1}, P_{2}$ have no common factors $x_{1}, x_{2}$. In such a case $\Omega(x)$ has a singular point $\left(x_{1}=0, x_{2}=a \neq 0\right)$ if $P_{2}(0, a)=0$, and $\left(x_{1}=a \neq 0, x_{2}=0\right)$ if $P_{1}(a, 0)=0$. It is straightforward to verify that vector fields $\Omega(z)$ and $\Omega(u)$ have the singular points $\left(z_{1}=0, z_{2}=0\right)$ and $\left(u_{1}=0, u_{2}=0\right)$ respectively. Vector fields $\Omega(x), \Omega(z)$ are equivalent everywhere except $x_{1} z_{1}=0, \Omega(x), \Omega(u)$ are equivalent everywhere except $x_{2} u_{2}=0\left(z_{1}=0, u_{2}=0\right.$ are the equators of the Poincaré sphere) and $\Omega(z)$, $\Omega(u)$ are equivalent everywhere except $z_{1}=0, u_{2}=0$; if $\Omega(z)$ has singular point $\left(z_{1}=0, z_{2}=a \neq 0\right)$, then $\Omega(u)$ has singular point $\left(u_{1}=1 / a \neq 0, u_{2}=0\right)$.

Without loss of generality we will suppose below that vector fields are continuously differentiable $\left(C^{1}\right)$ in both variables; we consider them to be defined in $\mathbf{R}_{+}^{2}$.

The objective of this work is to describe the asymptotic behavior of the orbits of the vector fields $\Omega(x), \Omega(z), \Omega(u)$ near the axes $x_{1} x_{2}=0$ and the equators of the Poincaré sphere $z_{1} u_{2}=0$.

To solve this problem we apply methods of the Newton diagram (polygon) (see [2]-7] and the references in [2]). We use a modification of the method, which has been developed in [8], 9], for an analysis of trajectories tending to a non-hyperbolic isolated equilibrium $O(0,0)$ with $t \rightarrow \infty$ and/or $t \rightarrow-\infty$ (the so-called $O$-orbits). In this paper we generalize these results analyzing isolated singular points (isp) $A\left(a_{1}, a_{2}\right)$ of vector fields $\Omega(x), \Omega(z), \Omega(u)$, for which $a_{1} a_{2}=0$. Note that this kind of problem still finds new applications (see, for example, [10], [11]).

\section{Newton polygon. The main statement}

2.1. Definitions. (See Figure 1.) (1) The set $\mathrm{M}=\left\{(u, \nu),\left|p_{\mu \nu}\right|+\left|q_{\mu \nu}\right| \neq 0\right\}$ is called the support of system (1.1) and of the vector field $\Omega(x)$, and $\left(p_{\mu \nu}, q_{\mu \nu}\right)$ is called the vector coefficient of the point $(\mu, \nu) \in \mathrm{M}$.

(2) The Newton polygon (NP) $\Gamma$ is the convex hull of M. $\Gamma$ consists of one vertex $\gamma=\gamma^{(0)}(\mu=0, \nu=0)$ or is the union of edges $\gamma=\gamma^{(1)} \in \Gamma$ together with their vertices.

(3) The edge $\gamma=\gamma^{(1)} \in \Gamma$ is called special and is denoted as $\gamma^{\mu}, \gamma^{\nu}, \gamma^{\mu^{0}}, \gamma^{\nu^{0}}$ if both of its vertices belong to either the $\mu$-axis or the $\nu$-axis, or the line $\mu=\mu^{0}$ or the line $\nu=\nu^{0}$, respectively; the edge $\gamma^{(1)}$ is called standard otherwise. Index $\alpha$ of an edge $\gamma$ is the number $\alpha=\alpha(\gamma)=\tan (\phi)$, where $\phi$ is the angle between the edge and the negative direction of the ordinate axis $\nu$; the special edges $\gamma^{\mu}, \gamma^{\mu^{0}}$ and $\gamma^{\nu}, \gamma^{\nu^{0}}$ have indexes $\alpha=0$ and $\alpha=\infty$ respectively.

(4) The vertex $\gamma \equiv \gamma^{(0)}(\mu, \nu)$ is called a boundary vertex if $\mu \nu=0$ and standard if not. The index $\beta$ of a vertex $\gamma$ is $\beta \equiv \beta(\gamma)=q_{\mu \nu} / p_{\mu \nu}$ if $p_{\mu \nu} \neq 0$ and $\beta=\infty$ if $p_{\mu \nu} \neq 0$.

(5) Inscribe $\Gamma$ into the rectangle $O_{00}(\mu=\nu=0), O_{\infty 0}\left(\mu^{0}, 0\right), O_{0 \infty}\left(0, \nu^{0}\right)$, $O_{\infty \infty}\left(\mu^{0}, \nu^{0}\right)$, where $\mu^{0}=\max _{\mu}(\mu,(\mu, \nu) \in \mathrm{M}), \nu^{0}=\max _{\nu}(\nu,(\mu, \nu) \in \mathrm{M})$. We call the Newton diagrams (ND) $\Gamma_{00}, \Gamma_{\infty 0}, \Gamma_{0 \infty}, \Gamma_{\infty \infty}$ the parts of $\Gamma$ which are visible from the corners $O_{00}, O_{\infty 0}, O_{0 \infty}, O_{\infty \infty}$ respectively. Thus $\Gamma=\Gamma_{00} \cup \Gamma_{\infty 0} \cup$ $\Gamma_{0 \infty} \cup \Gamma_{\infty \infty} \cup \gamma^{\mu} \cup \gamma^{\nu} \cup \gamma^{\mu^{0}} \cup \gamma^{\nu^{0}}$. Denote coherent sets $\mathrm{M}_{\Gamma}=\mathrm{M} \cap \Gamma, \mathrm{M}_{\Gamma_{0,0}}=\mathrm{M} \cap \Gamma_{00}$, $\mathrm{M}_{\Gamma_{\infty 0}}=\mathrm{M} \cap \Gamma_{\infty 0}, \mathrm{M}_{\Gamma_{\infty} \infty}=\mathrm{M} \cap \Gamma_{\infty \infty}, \mathrm{M}_{\Gamma_{0 \infty}}=\mathrm{M} \cap \Gamma_{0 \infty}, \mathrm{M} \cap \gamma$, where $\gamma$ is an edge or a vertex of $\Gamma$. Vector fields $\Omega_{\Gamma}(x), \Omega_{\Gamma_{00}}(x), \Omega_{\Gamma_{\infty 0}}(x), \Omega_{\Gamma_{\infty} \infty}(x), \Omega_{\Gamma_{0 \infty}}(x)$ and $\Omega_{\gamma}(x)$ are called the truncations of $\Omega(x)$ (and system (1.1)) onto the corresponding sets. In particular, $\Omega_{\Gamma}(x)$ is a vector field with fixed Newton polygon $\Gamma ; \Omega_{\Gamma_{00}}(x)$ is 
a vector field with fixed Newton diagram $\Gamma_{00}$. Let $\Omega(x) \equiv \Omega\left(X_{1}, X_{2}\right)$ be defined by systems (1.1), (1.2), which is presented in the form

$$
\begin{aligned}
\frac{d x_{1}}{d t} & =X_{1}\left(x_{1}, x_{2}\right) \equiv x_{1}\left(P_{1}^{\gamma}\left(x_{1}, x_{2}\right)+\phi_{1}\left(x_{1}, x_{2}\right)\right) \\
\frac{d x_{2}}{d t} & =X_{2}\left(x_{1}, x_{2}\right) \equiv x_{2}\left(P_{2}^{\gamma}\left(x_{1}, x_{2}\right)+\phi_{2}\left(x_{1}, x_{2}\right)\right) \\
P_{1}^{\gamma} & =\sum_{(\mu, \nu) \in \mathrm{M}_{\gamma}} p_{\mu \nu} x_{1}^{\mu} x_{2}^{\nu}, \quad P_{2}^{\gamma}=\sum_{(\mu, \nu) \in \mathrm{M}_{\gamma}} q_{\mu \nu} x_{1}^{\mu} x_{2}^{\nu} ; \\
\phi_{1} & =\sum_{(\mu, \nu) \in \mathrm{M}-\mathrm{M}_{\gamma}} p_{\mu \nu} x_{1}^{\mu} x_{2}^{\nu}, \quad \phi_{2}=\sum_{(\mu, \nu) \in \mathrm{M}-\mathrm{M}_{\gamma}} q_{\mu \nu} x_{1}^{\mu} x_{2}^{\nu} .
\end{aligned}
$$

Then the truncated vector field is $\Omega_{\gamma}(x) \equiv \Omega_{\gamma}\left(X_{1}^{\gamma}, X_{2}^{\gamma}\right)$, where $X_{1}^{\gamma} \equiv x_{1} P_{1}^{\gamma}\left(x_{1}, x_{2}\right)$, $X_{2}^{\gamma} \equiv x_{2} P_{2}^{\gamma}\left(x_{1}, x_{2}\right)$.

(a)

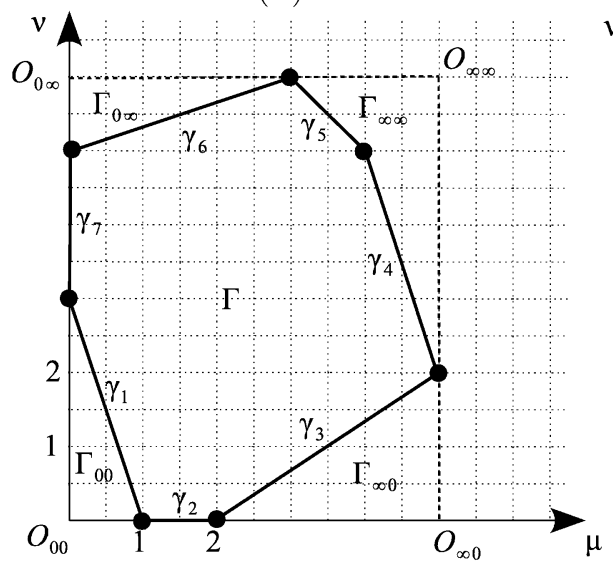

(b)

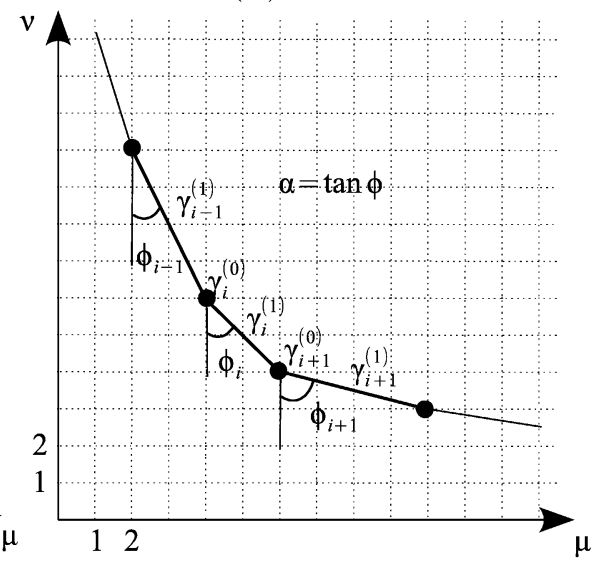

Figure 1. (a) $\Gamma$ is the Newton polygon of the system $x_{1}^{\prime}=x_{1} P_{1}\left(x_{1}, x_{2}\right), x_{2}^{\prime}=x_{2} P_{2}\left(x_{1}, x_{2}\right)$, where $P_{1}\left(x_{1}, x_{2}\right)=$ $p_{10} x_{1}+p_{20} x_{1}^{2}+p_{52} x_{1}^{5} x_{2}^{2}+p_{45} x_{1}^{4} x_{2}^{5}+p_{36} x_{1}^{3} x_{2}^{6}+p_{05} x_{2}^{5}+$ $p_{03} x_{2}^{3}, \quad P_{2}\left(x_{1}, x_{2}\right)=q_{10} x_{1}+q_{20} x_{1}^{2}+q_{52} x_{1}^{5} x_{2}^{2}+q_{45} x_{1}^{4} x_{2}^{5}+$ $q_{36} x_{1}^{3} x_{2}^{6}+q_{05} x_{2}^{5}+q_{03} x_{2}^{3}$. Support $\mathrm{M}=\mathrm{M}_{\Gamma}$ consists of points $(1,0),(2,0),(5,2),(4,5),(3,6),(0,5),(0,3) ; \Gamma=\Gamma_{00} \cup \Gamma_{\infty 0} \cup \Gamma_{0 \infty} \cup$ $\Gamma_{\infty} \cup \gamma^{\mu} \cup \gamma^{\nu}$, where $\Gamma_{00} \equiv \gamma_{1}$ with index $\alpha_{1}=1 / 3, \Gamma_{\infty 0} \equiv \gamma_{3}$ with index $\alpha_{3}=-3 / 2, \Gamma_{0 \infty} \equiv \gamma_{6}$ with index $\alpha_{6}=-3, \Gamma_{\infty \infty} \equiv \gamma_{4} \cup \gamma_{5}$, $\left(\alpha_{4}=1 / 3, \alpha_{5}=1\right)$ and $\gamma^{\mu}=\gamma_{7},\left(\alpha_{7}=0\right), \gamma^{\nu}=\gamma_{2},\left(\alpha_{2}=\infty\right)$ are special edges.

(b) A part of Newton diagram $\Gamma_{00}$ containing consequent edges $\gamma_{i-1}^{(1)}, \gamma_{i}^{(1)}, \gamma_{i+1}^{(1)}$ and edge $\gamma_{i}^{(1)}$ is bounded by vertices $\gamma_{i}^{(0)}\left(\mu_{i}, \nu_{i}\right)$, $\gamma_{i+1}^{(0)}\left(\mu_{i+1}, \nu_{i+1}\right)$. 
If $\gamma$ is a vertex $(\mu, \nu)$, a special edge, say $\gamma=\gamma^{\mu}$, or a standard edge $\gamma^{\alpha}$ with index $\alpha$, then

$$
\begin{gathered}
P_{1}^{\gamma}\left(x_{1}, x_{2}\right)=p_{\mu_{\gamma} \nu_{\gamma}} x_{1}^{\mu_{\gamma}} x_{2}^{\nu_{\gamma}}, \quad P_{2}^{\gamma}\left(x_{1}, x_{2}\right)=q_{\mu_{\gamma} \nu_{\gamma}} x_{1}^{\mu_{\gamma}} x_{2}^{\nu_{\gamma}} \\
P_{1}^{\gamma}\left(x_{1}, x_{2}\right)=x_{1}^{\mu} \sum_{\nu} p_{\mu \nu} x_{2}^{\nu}, \quad P_{2}^{\gamma}\left(x_{1}, x_{2}\right)=x_{1}^{\mu} \sum_{\nu} q_{\mu \nu} x_{2}^{\nu}, \quad(\mu, \nu) \in M_{\gamma}
\end{gathered}
$$

$$
P_{1}^{\gamma}\left(x_{1}, x_{2}\right)=\sum_{\mu+\alpha \nu=\sigma_{\gamma}} p_{\mu \nu} x_{1}^{\mu} x_{2}^{\nu}, \quad P_{2}^{\gamma}\left(x_{1}, x_{2}\right)=\sum_{\mu+\alpha \nu=\sigma_{\gamma}} q_{\mu \nu} x_{1}^{\mu} x_{2}^{\nu}, \quad \sigma_{\gamma} \equiv \text { const },
$$

respectively. Thus, a truncated vector field $\Omega_{\gamma}(x)$ is defined by the integrable system (2.1).

Definition (6). The vector field $\Omega_{\Gamma}(x)\left(\Omega_{\Gamma_{\infty}}(x), \Omega_{\Gamma_{\infty}}(x), \Omega_{\Gamma_{\infty} \infty}(x), \Omega_{\Gamma_{0 \infty}}(x)\right.$, $\left.\Omega_{\gamma}(x)\right)$ is called $\Gamma\left(\Gamma_{00}, \Gamma_{\infty 0}, \Gamma_{0 \infty}, \Gamma_{\infty \infty}, \gamma\right)$-non-degenerate with respect to isp $A\left(a_{1}, a_{2}\right)$, where $a_{1} a_{2}=0$ in one of the $x, z, u$-coordinates, if the following statements are fulfilled: (i) index $\beta$ of any interior vertex of $\Gamma\left(\Gamma_{00}, \Gamma_{\infty 0}, \Gamma_{0 \infty}, \Gamma_{\infty \infty}\right)$ is not equal to the indexes of the adjacent edges, and index $\beta$ of any boundary vertex is not equal to $\alpha=0, \infty$; (ii) for any edge $\gamma$ (having index $\alpha$ ) functions $P_{1}^{\alpha}(1, \omega), P_{2}^{\alpha}(1, \omega)$ have no common non-zero roots; (iii) the function $F^{\alpha}(\omega)=-\alpha P_{1}^{\alpha}(1, \omega)+P_{2}^{\alpha}(1, \omega)$ has no multiple non-zero roots.

According to the definition of non-degeneracy we can state

Proposition 2.1. Among all vector fields $\Omega(\Gamma)$ with the same Newton polygon $\Gamma$ the set of $\Gamma$-non-degenerate vector fields is open and dense.

\subsection{Asymptotics to equilibria.}

Theorem A. (1) Let $A\left(a_{1}, a_{2}\right)$ be a singular point of the $\Gamma$-non-degenerate vector field $\Omega(\nu)$ given in the Poincaré sphere by systems (1.1)-(1.2), (1.1)-(1.4) or (1.1)(1.6), where $a_{1} a_{2}=0$ in coordinates $\left(\nu_{1}, \nu_{2}\right)=\left(x_{1}, x_{2}\right)$ or $\left(\nu_{1}, \nu_{2}\right)=\left(z_{1}, z_{2}\right)$ or $\left(\nu_{1}, \nu_{2}\right)=\left(u_{1}, u_{2}\right)$. Any orbit of $\Omega(\nu)$ which tends to $A$ for $t \rightarrow \infty$ or $t \rightarrow-\infty$ in the phase coordinates $\left(\nu_{1}, \nu_{2}\right)$ has either power or trivial asymptotics; i.e., it can be presented in the form

$$
\nu_{2}-a_{2}=k \nu_{1}^{\rho}(1+o(1)), \quad \nu_{1}-a_{1}=\tilde{k} \nu_{2}^{\tilde{\rho}}(1+o(1)),
$$

where $\left(a_{2}, \rho \geq 0\right),\left(a_{1}, \tilde{\rho} \geq 0\right)$, constant $k \neq 0$ if $\rho>0, \tilde{k} \neq 0$ if $\tilde{p}>0$.

(2) Let $O(0,0)$ be a singular point of the $\Gamma$-non-degenerate vector field $\Omega(\nu)$ (i.e., $\left.a_{1}=a_{2}=0\right)$. Then $\tilde{\rho} \rho=1$ in (2.5). In $x$-coordinates the exponent $\rho$ coincides with index $\alpha$ of one of the edges $\gamma^{(1)} \in \Gamma$ or coincides with index $\beta$ of one of the vertices $\gamma^{(0)} \in \Gamma ; \rho=\alpha$ if the function $F^{\alpha}(\omega)=-\alpha P_{1}^{\alpha}(1, \omega)+P_{2}^{\alpha}(1, \omega)$ has root $\omega=k \neq 0, \rho=\beta$ if the vertex is interior and $\beta$ belongs to an interval composed by indexes of edges that are adjacent to this vertex.

The rest of the paper is organized as follows. In Section 3 we show the connections between power changes of variables in system (1.1), (1.2) and transformations of its Newton polygon. In Section 4 we provide a complete proof of Theorem A. 


\section{Power substitutions in System (1.1) AND TRANSFORMATIONS OF NP}

3.1. Auxiliary statements. Let us consider the question of how the change of the variables in systems (1.1), (1.2) affects NP $\Gamma$. Denote $\bar{\Gamma}$ as the transformed NP $\Gamma, \bar{\Omega}_{\Gamma} \equiv \Omega_{\bar{\Gamma}}$ the transformed vector field $\Omega_{\Gamma}$, etc., keeping the "initial" denotation supplemented by the bar. The following statement holds:

Proposition 3.1. The change of the independent variable in system (1.1),

$$
d t=x_{1}^{l_{1}} x_{2}^{l_{2}} d \tau, \quad l_{1}, l_{2} \in \mathbf{R},
$$

induces the linear transformation $\mathbf{L}: \Gamma \rightarrow \bar{\Gamma}$ shifting by the vector $\left(l_{1}, l_{2}\right)$ in the plane $(\mu, \nu) . \mathbf{L}$ is invertible and $\mathbf{L}^{-1}: \bar{\Gamma} \rightarrow \Gamma$ corresponds to the change $d \tau=$ $x_{1}^{-l_{1}} x_{2}^{-l_{2}} d t$.

Consider the power change of the variables in system (1.1):

$\left(x_{1}, x_{2}\right)=\left(\xi_{1}, \xi_{2}\right)^{E}: x_{1}=\xi_{1}^{e_{1}} \xi_{2}^{e_{2}}, x_{2}=\xi_{1}^{e_{3}} \xi_{2}^{e_{4}}, E=\left(\begin{array}{cc}e_{1} & e_{2} \\ e_{3} & e_{4}\end{array}\right), \Delta=\operatorname{det}(E)\left(x_{1}, x_{2}\right) \in \mathbf{R}_{2}^{+}$.

If $\Delta \neq 0$, then (3.2) has the inverse $\left(\xi_{1}, \xi_{2}\right)=\left(x_{1}, x_{2}\right)^{E^{-1}}$ and converts (1.1) to the form:

$$
\begin{aligned}
& \frac{d \xi_{1}}{d \tau}=\Xi_{1}\left(\xi_{1}, \xi_{2}\right) \equiv \xi_{1} H_{1}\left(\xi_{1}, \xi_{2}\right)=\xi_{1}\left(e_{4} P_{1}\left(x_{1}, x_{2}\right)-e_{2} P_{2}\left(x_{1}, x_{2}\right)\right) \equiv \xi_{1} \sum_{\bar{\mu}, \bar{\nu}} p_{\overline{\mu \nu}} \xi_{1}^{\bar{\mu}} \xi_{2}^{\bar{\nu}} \\
& \frac{d \xi_{2}}{d \tau}=\Xi_{2}\left(\xi_{1}, \xi_{2}\right) \equiv \xi_{2} H_{2}\left(\xi_{1}, \xi_{2}\right)=\xi_{2}\left(-e_{3} P_{1}\left(x_{1}, x_{2}\right)+e_{1} P_{2}\left(x_{1}, x_{2}\right)\right) \equiv \xi_{2} \sum_{\bar{\mu}, \bar{\nu}} q_{\overline{\mu \nu}} \xi_{1}^{\bar{\mu}} \xi_{2}^{\bar{\nu}} \\
& \bar{p}_{\mu \nu} \equiv p_{\overline{\mu \nu}}=e_{4} p_{\mu \nu}-e_{2} q_{\mu \nu}, \quad \bar{q}_{\mu \nu} \equiv q_{\overline{\mu \nu}}=-e_{3} p_{\mu \nu}+e_{1} q_{\mu \nu} .
\end{aligned}
$$

Let $\overline{\mathrm{M}}$ be the support of system (3.3). Comparing powers $(\mu, \nu),(\bar{\mu}, \bar{\nu})$ of systems (1.1), (1.2) and (3.3) we see that the change (3.2) induces a linear transformation $\mathbf{G}: \mathrm{M} \rightarrow \overline{\mathrm{M}}$ with the matrix $E^{T}:(\mu, \nu) \rightarrow(\bar{\mu}, \bar{\nu}): \bar{\mu}=e_{1} \mu+e_{3} \nu, \bar{\nu}=e_{2} \mu+e_{4} \nu$. If $\Delta \neq 0$, then inverse to $\mathbf{G}$ is given by $\mathbf{G}^{-1}$, which has the matrix

$$
\left(E^{T}\right)^{-1}=\frac{1}{\Delta}\left(\begin{array}{cc}
e_{4} & -e_{2} \\
-e_{3} & e_{1}
\end{array}\right):(\bar{\mu}, \bar{\nu})=\mathbf{G}^{-1}(\mu, \nu) ;
$$

$\mathbf{G}^{-1}$ transforms the Newton polygon $\Gamma \rightarrow \bar{\Gamma}$ such that the vector fields $\Omega_{\Gamma}$ and $\bar{\Omega}_{\Gamma} \equiv$ $\Omega_{\bar{\Gamma}}$ completely define one another. The systems can be non-equivalent only at their equilibria, in particular at the coordinate axes. To avoid this "non-equivalency" we use the operators $\mathbf{L} \circ \mathbf{G}$ and $(\mathbf{L} \circ \mathbf{G})^{-1}$.

Lemma 3.1 ([8, 9]). (1) The indexes of the vertices and edges of $\bar{\Gamma}$, which are obtained from $\Gamma$ by the transformation $\mathbf{L}$, are identical to those of $\Gamma$.

(2) If $\Delta \neq 0$, then the transformation $\mathbf{G}^{-1}: \Gamma_{\Omega(x)} \rightarrow \Gamma_{\bar{\Omega}(\xi)}$ converts any edge and any vertex of $\Gamma_{\Omega(x)}$ into the edge and the vertex of $\Gamma_{\bar{\Omega}(\xi)}$. Indexes $\alpha, \bar{\alpha}$ of the edges $\gamma^{(1)}, \bar{\gamma}^{(1)}$ and $\beta, \bar{\beta}$ of vertices $\gamma^{(0)}, \bar{\gamma}^{(0)}$ are expressed by the formulas

$$
\bar{\alpha}=\left(\alpha e_{1}-e_{3}\right) /\left(e_{4}-\alpha e_{2}\right), \quad \bar{\beta}=\left(\beta e_{1}-e_{3}\right) /\left(e_{4}-\beta e_{2}\right) .
$$




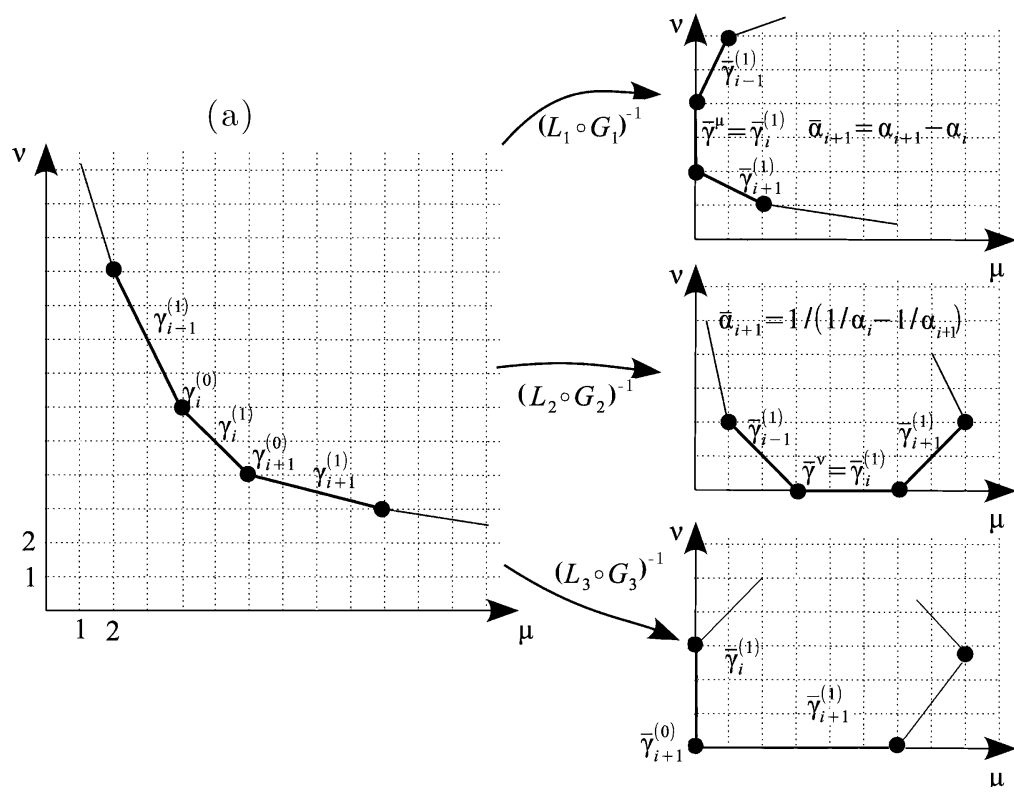

(b)

(c)

Figure 2. (a) A part of Newton diagram $\Gamma_{00}$ (see Figure 1(b)); (b-d) results of changes of variables $\left(x_{1}, x_{2}\right)=\left(\xi_{1}, \xi_{2}\right)^{E}$, where

$$
\begin{gathered}
E=\left(\begin{array}{cc}
1 & 0 \\
\alpha_{i} & 1
\end{array}\right)(\mathrm{b}), \quad E=\left(\begin{array}{cc}
1 & 1 / \alpha_{i} \\
0 & 1
\end{array}\right)(\mathrm{c}), \\
E=\left(\begin{array}{cc}
1 & 1 /\left(\alpha_{i+1}-\alpha_{i}\right) \\
\alpha_{i} & \alpha_{i+1} /\left(\alpha_{i+1}-\alpha_{i}\right)
\end{array}\right)(\mathrm{d})
\end{gathered}
$$

edges $\gamma_{i}^{(1)} \in \Gamma_{00} \subset \Gamma \rightarrow \gamma^{\mu} \in \bar{\Gamma}(\mathrm{b}), \gamma_{i}^{(1)} \in \Gamma_{00} \subset \Gamma \rightarrow \gamma^{\nu} \in \bar{\Gamma}$ (c), and the vertex $\gamma_{i+1}^{(0)} \rightarrow \bar{\gamma}_{i+1}^{(0)}(0,0) \in \bar{\Gamma}_{00}(\mathrm{~d})$.

Important applications of Lemma 3.1 are given by the following statement.

Proposition 3.2. (1) Let $\gamma^{(1)} \in \Gamma_{00}$ be a standard edge with index $\alpha$. The linear transformation $\left(\mathbf{L}_{1} \circ \mathbf{G}_{1}\right)^{-1}$ with matrix $E_{1}=\left(\begin{array}{ll}1 & 0 \\ \alpha & 1\end{array}\right)$ and $\left(l_{1}, l_{2}\right)=(\mu+\alpha \nu, 0)$, $(\mu, \nu) \in \mathbf{M}_{\gamma}$ converts $\gamma^{(1)}$ into the special edge $\bar{\gamma}^{\mu}$ (see Figure $2(a),(b)$ ). The linear transformation $\left(\mathbf{L}_{2} \circ \mathbf{G}_{2}\right)^{-1}$ with matrix $E_{2}=\left(\begin{array}{cc}1 & 1 / \alpha \\ 0 & 1\end{array}\right)$ and $\left(l_{1}, l_{2}\right)=(0,(\mu+\alpha \nu) / \alpha)$, $(\mu, \nu) \in \mathbf{M}_{\gamma}$ converts $\gamma^{(1)}$ into the special edge $\bar{\gamma}^{\nu}$ (see Figure $2(a),(c)$ ).

(2) Let $\gamma^{(0)}(\mu, \nu) \in \Gamma_{00}$ be an interior vertex, which is common for edges $\gamma_{1}^{(1)}, \gamma_{2}^{(1)}$ (having indexes $\left.0<\alpha_{1}<\alpha_{2}\right)$. The linear transformation $\left(\mathbf{L}_{3} \circ \mathbf{G}_{3}\right)^{-1}$ with matrix

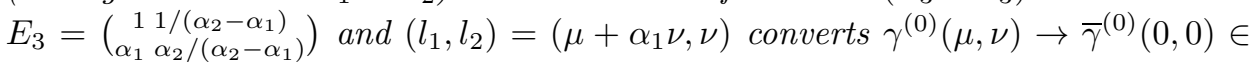
$\bar{\Gamma}_{00}$ such that $\bar{\Gamma}_{00}$ consists of only this vertex (see Figure $2(a),(d)$ ).

3.2. Newton diagrams and Poincaré coordinates. Poincaré changes (1.3) and (1.5) are particular cases of changes (3.2) with $E_{1}=\left(\begin{array}{ll}-1 & 0 \\ -1 & 1\end{array}\right)$ and $E_{2}=\left(\begin{array}{ll}1 & -1 \\ 0 & -1\end{array}\right)$, respectively. Supplemented by the change of independent variable (3.1), where $l_{1}=\sigma, l_{2}=0$ for $\mathbf{L}_{1}$ and $l_{2}=\sigma, l_{1}=0$ for $\mathbf{L}_{2}$ with $\sigma \equiv \max _{(\mu, \nu) \in \mathbf{M}_{\Gamma}}(\mu+\nu)$, these changes transform the vector field $\Omega(x)$ defined by system (1.1), (1.2) to the vector 
fields $\Omega(z)$ and $\Omega(u)$ defined by systems (1.1), (1.4) and (1.1), (1.5) respectively. Let $\Gamma$ be a Newton polygon of $(1.1),(1.2)$ and $\bar{\Gamma}^{1}, \bar{\Gamma}^{2}$ be Newton polygons of after transformations $\left(\mathbf{L}_{1} \circ \mathbf{G}_{1}\right)^{-1}$ with $E=E_{1}$ and $\left(\mathbf{L}_{2} \circ \mathbf{G}_{2}\right)^{-1}$ with $E=E_{2}$, and let $\bar{\Gamma}_{00}^{1} \in \bar{\Gamma}^{1}, \bar{\Gamma}_{00}^{2} \in \bar{\Gamma}^{2}$ be their Newton diagrams. To find the "preimages" of $\bar{\Gamma}_{00}^{1}, \bar{\Gamma}_{00}^{2}$ under transformations $\mathbf{G}_{1}^{-1}, \mathbf{G}_{2}^{-1}$ we introduce coherent open polygons $\widehat{\Gamma}_{1}=\left(\Gamma_{\infty 0} \cup \gamma^{\mu^{0}} \cup \Gamma_{\infty \infty}^{\alpha<1}\right) \subset \Gamma, \widehat{\Gamma}_{2}=\left(\Gamma_{0 \infty} \cup \gamma^{\nu^{0}} \cup \Gamma_{\infty \infty}^{\alpha>1}\right) \subset \Gamma$ (see Figure 3(a), (b), (c)), where $\Gamma_{\infty \infty}^{\alpha<1}$ and $\Gamma_{\infty \infty}^{\alpha>1}$ are parts of $\Gamma_{\infty \infty}$ that contain edges $\gamma$ with indexes $\alpha<1$ and with indexes $\alpha>1$, respectively, or $\widehat{\Gamma}_{1}=\gamma^{(0)}=\widehat{\Gamma}_{2}$ if $\Gamma_{\infty 0}, \Gamma_{\infty \infty}, \Gamma_{0 \infty}$ consist of one point $\gamma^{(0)}$.

According to Lemma 3.1 we have from formula (3.4) that for $E=E_{1}$ and $E=E_{2}$, respectively,

$$
\begin{array}{cl}
\bar{\alpha}=1-\alpha, & \bar{\beta}=1-\beta, \\
\bar{\alpha}=\alpha /(\alpha-1), & \bar{\beta}=\beta /(\beta-1),
\end{array}
$$

where $\bar{\alpha} \equiv \alpha\left(\bar{\gamma}^{(1)} \in \bar{\Gamma}\right), \bar{\beta} \equiv \beta\left(\bar{\gamma}^{(0)} \in \bar{\Gamma}\right), \alpha \equiv \alpha(\gamma \in \Gamma), \beta \equiv \beta\left(\gamma^{(0)} \in \Gamma\right)$. After additional algebraic verifications we prove the following statement.

Theorem 3.1 (see Figure 3(a),(b),(c)). $\bar{\Gamma}_{00}$ is the one-to-one image of the union $\widehat{\Gamma}^{1}$ under transformation $\left(\mathbf{L}_{1} \circ \mathbf{G}_{1}\right)^{-1}$ and the union $\widehat{\Gamma}^{2}$ under transformation $\left(\mathbf{L}_{2} \circ\right.$ $\left.\mathbf{G}_{2}\right)^{-1}$; the edge $\gamma^{\alpha} \in \Gamma_{\infty \infty}$ with $\alpha=1$ transforms to the edge $\bar{\gamma}^{\mu} \in \bar{\Gamma}^{1}$ under $\left(\mathbf{L}_{1} \circ \mathbf{G}_{1}\right)^{-1}$ and to the edge $\bar{\gamma}^{\nu} \in \bar{\Gamma}^{2}$ under $\left(\mathbf{L}_{2} \circ \mathbf{G}_{2}\right)^{-1}$.

It is straightforward to verify the following statement, which describes the conditions of existence of isolated singular points $A\left(a_{1}, a_{2}\right), a_{1} a_{2}=0$ in the nondegenerate vector field $\Omega_{\Gamma}(x)$.

Proposition 3.3. Let $\Omega_{\Gamma}$ be a $\Gamma$-non-degenerate vector field given by (1.1). Then

(1) point $(0,0)$ is the isp of vector field $\Omega_{\Gamma}$ if and only if it is isp of $\Omega_{\Gamma_{00}}(\nu)$;

(2) point $A\left(0, a_{2}\right), a_{2} \neq 0\left(A\left(a_{1}, 0\right), a_{1} \neq 0\right)$ is the isp of $\Omega_{\Gamma}$ if and only if $\Gamma$ contains a special edge $\gamma^{\mu}\left(\gamma^{\nu}\right)$ such that the function $F^{\gamma}\left(x_{2}\right) \equiv P_{2}^{\gamma^{\mu}}\left(0, x_{2}\right)$ has a root $a_{2}\left(F^{\gamma^{\nu}}\left(x_{1}\right) \equiv P_{1}^{\gamma}\left(x_{1}, 0\right)\right)$ has a root $a_{1}$.

According to Theorem 3.1 analysis of all asymptotics of orbits of the $\Gamma$-nondegenerate vector field $\Omega_{\Gamma}$ is reduced to the analysis of asymptotics of orbits of the vector fields $\Omega_{\Gamma_{0,0}}$ and $\Omega_{\gamma^{\mu}}, \Omega_{\gamma}$.

\section{Proof of Theorem A. Description of asymptotics}

4.1. Andronov's statement. Orbits of the $\gamma$-non-degenerate vector field and its $\gamma$-truncation. Further, we use the statement, based on Lemmas 1 and 2 in Chapter IV of [1].

Lemma 4.1. Let $O(0,0)$ be an isolated simple equilibrium of the $C^{1}$-system (1.1), which has a triangular Jordan matrix at $O$ with non-zero eigenvalues $\lambda_{1}, \lambda_{2}$. If $\lambda_{1} \lambda_{2}>0$, then $O$-orbits of the vector field defined by this system have asymptotics $x_{2}=k x_{1}^{\lambda_{2} / \lambda_{1}}(1+o(1)), k \neq 0$. 
(a)

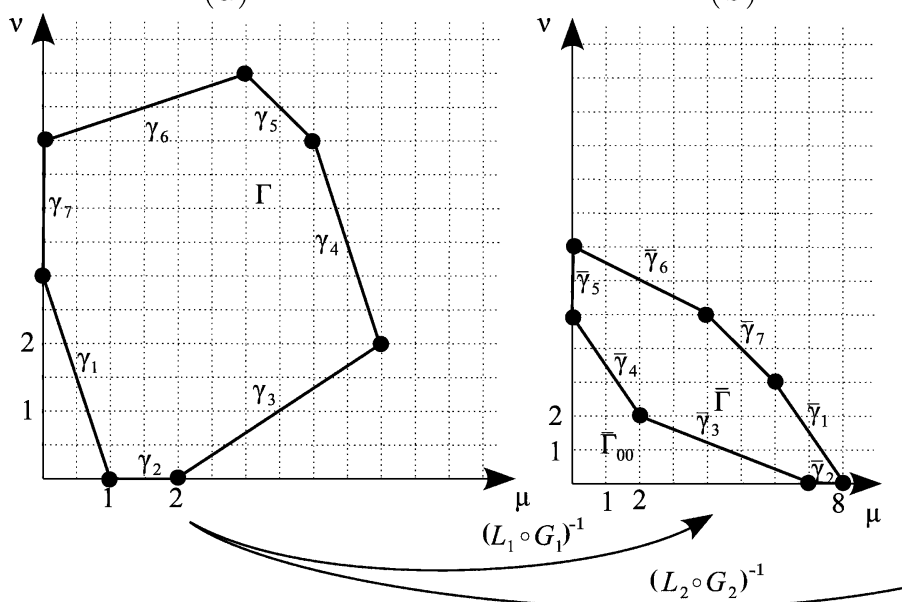

(c)

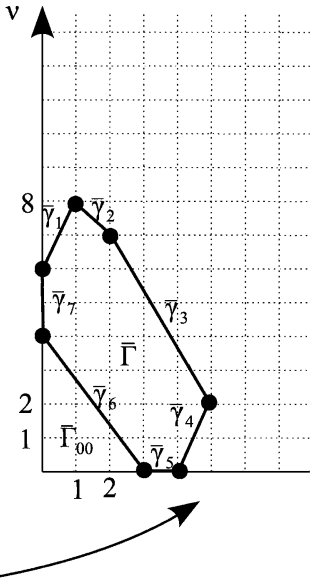

Figure 3. (a) Newton polygon $\Gamma$ of the vector field $\Omega_{\Gamma}(x)$ which is presented and described in Figure 1(a), polygons $\widehat{\Gamma}_{1}=\left(\gamma_{3} \cup \gamma_{4}\right)$, $\widehat{\Gamma}_{2}=\left(\gamma_{6} \cup \gamma_{7}\right)$, edge $\gamma_{5} \in \Gamma_{\infty \infty}, \alpha\left(\gamma_{5}\right)=1$; (b) Newton polygon $\bar{\Gamma}$ of the vector field $\bar{\Omega}_{\Gamma}(z) \equiv \Omega_{\bar{\Gamma}}(z)$ in the Poincaré coordinates $\left(z_{1}=\right.$ $\left.1 / x_{1}, z_{2}=x_{2} / x_{1}\right)$, polygon $\widehat{\Gamma}_{1} \in \Gamma \rightarrow\left(\bar{\gamma}_{3} \cup \bar{\gamma}_{4}\right) \equiv \bar{\Gamma}_{00} \in \bar{\Gamma}, \gamma_{5} \rightarrow$ $\gamma^{\mu} \in \bar{\Gamma}$; (c) Newton polygon $\bar{\Gamma}$ of the vector field $\bar{\Omega}_{\Gamma}(u) \equiv \Omega_{\bar{\Gamma}}(u)$ in the Poincaré coordinates $\left(u_{1}=x_{2} / x_{1}, u_{2}=1 / x_{2}\right)$, polygon $\widehat{\Gamma}_{2} \in \Gamma \rightarrow\left(\bar{\gamma}_{6} \cup \bar{\gamma}_{7}\right) \equiv \bar{\Gamma}_{00} \in \bar{\Gamma}$, edge $\gamma_{5} \rightarrow \gamma^{\nu} \in \bar{\Gamma}$.

Further, we consider systems (1.1), (1.2) in the form of (2.1):

$$
\begin{aligned}
& x_{1}^{\prime}=x_{1} P_{1}\left(x_{1}, x_{2}\right) \equiv x_{1}\left(P_{1}^{\gamma}\left(x_{1}, x_{2}\right)+\phi_{1}\left(x_{1}, x_{2}\right)\right), \\
& x_{2}^{\prime}=x_{2} P_{2}\left(x_{1}, x_{2}\right) \equiv x_{2}\left(P_{2}^{\gamma}\left(x_{1}, x_{2}\right)+\phi_{2}\left(x_{1}, x_{2}\right)\right),
\end{aligned}
$$

where $P_{1}^{\gamma}\left(x_{1}, x_{2}\right), P_{2}^{\gamma}\left(x_{1}, x_{2}\right), \phi_{1}\left(x_{1}, x_{2}\right), \phi_{2}\left(x_{1}, x_{2}\right)$ present specifying functions. Let $\gamma \equiv \gamma^{(1)} \in \Gamma_{00}$.

\subsection{Asymptotics of the orbits tending to an equilibrium with one zero coordinate.}

Proposition 4.1. Let the $\Gamma$-non-degenerate vector field $\Omega_{\Gamma}$ given by (4.1), (1.2), have an isolated singular point $A_{2}\left(x_{1}=0, x_{2}=a_{2} \neq 0\right)$ and/or $A_{1}\left(x_{1}=a_{1} \neq 0\right.$, $\left.x_{2}=0\right)$. Then orbits tending to $A_{2}\left(A_{1}\right)$ with $x_{1} \rightarrow 0\left(x_{2} \rightarrow 0\right)$ have power asymptotics $(2.5)$ or trivial $x_{1}=0, x_{2}=a_{2}$ and/or $x_{1}=a_{1}, x_{2}=0$.

Proof. Let us consider the first case: $P_{2}\left(0, a_{2}\right)=0, P_{1}\left(0, a_{2}\right) \neq 0$. According to Proposition 3.3, $\gamma \equiv \gamma^{\mu}, \alpha(\gamma)=0$, in (4.1) functions $P_{1}^{\gamma}\left(x_{1}, x_{2}\right)=\sum_{\nu} p_{\mu \nu} x_{2}^{\nu}$, $P_{2}^{\gamma}\left(x_{1}, x_{2}\right)=\sum_{\nu} q_{\mu \nu} x_{2}^{\nu}\left(P_{1}^{\gamma}, P_{2}^{\gamma}\right.$ are of the form $\left.(2.3)\right)$, and $\phi_{1}\left(0, x_{2}\right)=\phi_{2}\left(0, x_{2}\right)=0$ (see, for example, edge $\gamma_{7}$ in Figure 3(a)). Due to conditions of non-degeneracy (ii), (iii) (see Definition (6)), $a_{2}$ is a simple root of function $P_{2}^{\gamma^{\mu}}\left(0, x_{2}\right)$ and $P_{1}^{\gamma^{\mu}}\left(1, a_{2}\right) \neq 0$. Supposing that system (4.1) is continuously differential in both variables, we find eigenvalues at $\left(0, a_{2}\right): \lambda_{1}=P_{1}^{\gamma^{\mu}}\left(0, a_{2}\right), \lambda_{2}=a_{2} P_{2 x_{2}}^{\gamma^{\mu}}\left(0, a_{2}\right), \lambda_{1} \lambda_{2} \neq 0$. According to 
Lemma 4.1 the vector field defined by (4.1) has orbits of the form (2.5): $x_{2}-a_{2}=$ $k x_{1}^{\lambda_{2} / \lambda_{1}}(1+o(1))$, where $k$ is an arbitrary constant if $\lambda_{1} \lambda_{2}>0$ and $k=0$ if $\lambda_{1} \lambda_{2}<0$.

The second case can be considered in an analogous way. Proposition 4.1 is proven.

4.3. Asymptotics to $O$-orbits. The following theorem has been formulated and proven for polynomial vector fields in [8] (see also [7]). The case $\Gamma_{00}$ consisting of one standard edge was considered in 10 .

Theorem 4.1. (i) Asymptotics of O-orbits of the $\Gamma$-non-degenerate vector field $\Omega(x)$ coincide with the asymptotics of $O$-orbits of $\Omega_{\Gamma_{00}}(x)$ and have the form $(2.5)$ : $x_{2}=k x_{1}^{\rho}(1+o(1))$, where the exponent $\rho>0$ equals the index $\alpha$ of one of the edges $\gamma^{(1)} \in \Gamma_{00}$ or equals the index $\beta$ of one of the vertices $\gamma^{(0)} \in \Gamma_{00}$.

(ii) $\rho=\alpha$ if function $F^{\alpha}\left(\xi_{2}\right)=-\alpha P_{1}^{\alpha}\left(1, \xi_{2}\right)+P_{2}^{\alpha}\left(1, \xi_{2}\right)$ has a root $\xi_{2}=k \neq 0$, which serves the coefficient of $(2.5) ; \rho=\beta$ and coefficient $k \neq 0$ is any number if $\beta \in(\hat{\alpha}, \hat{\hat{\alpha}})$, where $0 \leq \hat{\alpha}<\hat{\hat{\alpha}} \leq \infty$ are indexes of edges that are adjacent to this vertex, $\hat{\alpha}=0$ if vertex $\gamma^{(0)}(0, \nu) \in \Gamma_{00}$ and/or $\hat{\hat{\alpha}}=\infty$ if vertex $\gamma^{(0)}(\mu, 0) \in \Gamma_{00}$.

Below we prove Theorem 4.1 under the assumption that point $O$ is the isp of the $\Gamma$-non-degenerate continuously differentiable vector field $\Omega(x)$ defined by (1.1), (1.2).

Proof of Theorem 4.1. (i) Let ND $\Gamma_{00}$ consist of one vertex $\gamma^{(0)}\left(\mu_{0}, \nu_{0}\right)$. Because $O(0,0)$ is an isolated point, $\mu=\mu_{0}=0, \nu=\nu_{0}=0$. Therefore system (4.1) is of the form (4.1), (2.2): $P_{1}^{\gamma}\left(x_{1}, x_{2}\right)=p_{00}=\lambda_{1}, P_{2}^{\gamma}\left(x_{1}, x_{2}\right)=q_{00}=\lambda_{2}$ such that $p_{00} q_{00} \neq 0, \phi_{1}\left(0, x_{2}\right)=\phi_{2}\left(x_{1}, 0\right)=0$. According to Lemma 4.1 the system has $O$-orbits in the form (2.5) with $\rho \equiv \beta_{0}=q_{00} / p_{00}$, where the coefficient $k$ is an arbitrary constant if $p_{00} q_{00}>0$ (i.e. $O$ is a node) and $k=0$ if $p_{00} q_{00}<0(O$ is a saddle whose separatrices are $x_{1}=0, x_{2} \neq 0$ and $\left.x_{2}=0, x_{1} \neq 0\right)$.

(ii) Let ND $\Gamma_{00}$ contain the unique edge $\gamma=\gamma^{(1)}$ (having index $\alpha$ ) which is bounded by vertices $\gamma_{1}^{(0)}\left(\mu_{1}, \nu_{1}\right), \gamma_{2}^{(0)}\left(\mu_{2}, \nu_{2}\right), \mu_{1}=0<\mu_{2}, \nu_{1}>\nu_{2}=0$, whose indexes are $\beta_{1}, \beta_{2}$. Then system (4.1) is of the form (2.1), (2.4), where $P_{1}^{\alpha}\left(x_{1}, x_{2}\right)=$ $\sum_{\mu+\alpha \nu=\sigma} p_{\mu \nu} x_{1}^{\mu} x_{2}^{\nu}, P_{2}^{\alpha}\left(x_{1}, x_{2}\right)=\sum_{\mu+\alpha \nu=\sigma} q_{\mu \nu} x_{1}^{\mu} x_{2}^{\nu}$.

Due to Proposition 3.2, change of variables (3.2): $x_{1}=\xi_{1}, x_{2}=\xi_{1}^{\alpha} \xi_{2}$, supplemented by $(3.1)$ with $\left(l_{1}, l_{2}\right)=\left(\mu_{2}, 0\right)$, converts system (4.1) to the system with

$$
\begin{gathered}
\bar{P}_{1}\left(\xi_{1}, \xi_{2}\right)=P_{1}^{\alpha}\left(1, \xi_{2}\right)+\bar{\phi}_{1}\left(\xi_{1}, \xi_{2}\right), \quad \bar{P}_{2}\left(\xi_{1}, \xi_{2}\right)=F^{\alpha}\left(\xi_{2}\right)+\bar{\phi}_{2}\left(\xi_{1}, \xi_{2}\right), \\
\bar{\phi}_{1}\left(0, \xi_{2}\right)=\bar{\phi}_{2}\left(0, \xi_{2}\right)=0, \quad F^{\alpha}\left(\xi_{2}\right)=-\alpha P_{1}^{\alpha}\left(1, \xi_{2}\right)+P_{2}^{\alpha}\left(1, \xi_{2}\right) .
\end{gathered}
$$

With this change the edge $\gamma^{(1)} \in \Gamma_{00} \subset \Gamma$ is transformed into the special edge $\bar{\gamma}^{\mu} \in \bar{\Gamma}$ (see Figure 2(a),(b)), vertex $\gamma_{2}^{(0)}\left(\mu_{2}, 0\right) \rightarrow \bar{\gamma}_{2}^{(0)}(0,0) \in \bar{\Gamma}_{00}$, where $\bar{\gamma}_{00}^{(0)}$ is the unique vertex of $\bar{\Gamma}_{00}$, and $\bar{\beta}=\beta_{2}-\alpha$ is the index of $\bar{\gamma}_{00}^{(0)}$. According to Proposition 4.1 and conditions of non-degeneracy (ii), (iii) (see Definition (6)), if $F^{\alpha}\left(\xi_{2}\right)$ has root $\xi_{2}=a_{2} \neq 0$, then the system (3.3) has an orbit with asymptotics $\xi_{2}-a_{2}=o(1), o(1) \rightarrow 0$ for $\xi_{1} \rightarrow 0$. In such a case system (4.1) has an orbit with power asymptotics $x_{2}=a_{2} x_{1}^{\alpha}(1+o(1))$. The vertex $\bar{\gamma}_{00}^{(0)} \in \bar{\Gamma}_{00}$ has index $\bar{\beta} \equiv \bar{q}_{00} / \bar{p}_{00} \neq 0, \infty$, where $\bar{p}_{00}=P_{1}^{\alpha}(1,0)=p_{\mu_{2} 0}, \bar{q}_{00}=F^{\alpha}(0)=-\alpha p_{\mu_{2} 0}+q_{\mu_{2} 0}$ (see Figure 2(a),(d)). Therefore, we have the case which has been discussed in part (i): system (3.3) has an orbit with asymptotics $\xi_{2}=k \xi_{1}^{\bar{\beta}}(1+o(1))$ or trivial $\xi_{2}=0$. In these variables $\left(x_{1}, x_{2}\right)$ these asymptotics obtain the form $x_{2}=k x_{1}^{\beta}(1+o(1))$. 
The second change of variables (3.2): $x_{1}=\xi_{1} \xi_{1}^{1 / \alpha}, x_{2}=\xi_{2}$, supplemented by (3.1) with $\left(l_{1}, l_{2}\right)=\left(0, \nu_{1}\right)$, lead to the transformation of edge $\gamma^{(1)}$ to the special edge $\bar{\gamma}^{\nu}$ (see Figure 2(a),(c),(d)) such that vertex $\gamma_{1}^{(0)}\left(0, \nu_{1}\right) \rightarrow \bar{\gamma}_{00}^{(0)} \in \bar{\Gamma}_{00}$, where

$\bar{\gamma}_{00}^{(0)}$ is the unique vertex of $\bar{\Gamma}_{00}$. Due to conditions of non-degeneracy: $\beta_{1} \neq \alpha, \infty$, index of $\bar{\gamma}_{00}^{(0)}: \bar{\beta}=\beta_{1}-\alpha \neq 0, \infty$. Repeating the above arguments we finish the proof of statements considering the case.

Actually, we have proven that $O$-orbits of the $\Gamma_{00}$-non-degenerated vector field $\Omega_{\Gamma}$ can have only power or trivial asymptotics and described exponents and coefficients of these asymptotics in the form given in Theorem 4.1.

(iii) Note now that according to Lemma 3.1 and Proposition 3.2 any system (4.1) whose ND $\Gamma_{00}$ contains $N>1$ standard edges by changes of variables (3.2) and (3.1) can be reduced to the system whose ND $\bar{\Gamma}_{00}$ contains the only standard edge, and the "new" system will be $\bar{\Gamma}_{00}$-non-degenerate if the initial one was $\Gamma_{00^{-}}$ non-degenerate. Thus, all asymptotics of $O$-orbits are the same as described in Theorem 4.1. Theorem 4.1 is proven.

(iv) Taking into consideration the statements of Theorem 3.1, we can state that orbits tending to singular points of vector fields defined by systems (1.1), (1.4) and (1.6) have power asymptotics as well. For example, let an orbit of system (1.4) be of the form $z_{2}=k z_{1}^{\bar{\alpha}}(1+o(1))$; i.e., $\bar{\alpha}=\alpha(\bar{\gamma}), \bar{\gamma} \in \bar{\Gamma}_{00}$ and $k \neq 0$ is a root of the function $F^{\bar{\alpha}}(\omega)$. Due to (1.5) and (3.4) $x_{2}=k x_{1}^{1-\bar{\alpha}}(1+o(1))=k x_{1}^{\alpha}(1+o(1))$, where $\alpha=\alpha(\gamma)$ and $\gamma$ is the preimage of edge $\bar{\gamma}$ under the transformation with matrix $E_{1}$ (see (3.3)). In an analogous way one can verify other cases and show that exponents and coefficients of the mentioned asymptotics are expressed in terms of indexes of vertices and edges of the Newton polygon. This finishes the proof of Theorem A.

\section{ACKNOWLEDGEMENT}

The author is grateful to the anonymous referee for comments that helped to improve this manuscript and figures.

\section{REFERENCES}

[1] A. A. Andronov, E. A. Leontovich, I. I. Gordon, and A. G. Maŭer, Qualitative theory of second-order dynamic systems, Halsted Press (A division of John Wiley \& Sons), New YorkToronto, Ont., 1973. Translated from the Russian by D. Louvish. MR0350126 (50:2619)

[2] Alexander D. Bruno, Power geometry in algebraic and differential equations, North-Holland Mathematical Library, vol. 57, North-Holland Publishing Co., Amsterdam, 2000. Translated from the 1998 Russian original by V. P. Varin and revised by the author. MR 1773512 (2002c:37071)

[3] Max Frommer, Die Integralkurven einer gewöhnlichen Differentialgleichung erster Ordnung in der Umgebung rationaler Unbestimmtheitsstellen (German), Math. Ann. 99 (1928), no. 1, 222-272, DOI 10.1007/BF01459096. MR.1512449

[4] D. N. Bernstein, A. G. Kušnirenko, and A. G. Hovanskiı̌, Newton polyhedra (Russian), Uspehi Mat. Nauk 31 (1976), no. 3(189), 201-202. MR0492376 (58:11500)

[5] Freddy Dumortier, Singularities of vector fields, Monografías de Matemática [Mathematical Monographs], vol. 32, Instituto de Matemática Pura e Aplicada, Rio de Janeiro, 1978. MR526571 (81k:58050)

[6] Shui-Nee Chow and Jack K. Hale, Methods of bifurcation theory, Grundlehren der Mathematischen Wissenschaften [Fundamental Principles of Mathematical Science], vol. 251, SpringerVerlag, New York, 1982. MR660633(84e:58019) 
[7] V. I. Arnol'd and Yu. S. Il'yashenko, Ordinary differential equations (Russian), Current problems in mathematics. Fundamental directions, Vol. 1, Itogi Nauki i Tekhniki, Akad. Nauk SSSR, Vsesoyuz. Inst. Nauchn. i Tekhn. Inform., Moscow, 1985, pp. 7-149, 244. MR823489 (87e:34049)

[8] F. S. Berezovskaya, Analysis of multiple equilibrium of a plane differential equation system with application of Newton diagram, Ph.D. Thesis, Puschino, 1977.

[9] F. S. Berezovskaya and N. B. Medvedeva, A complicated singular point of "center-focus" type and the Newton diagram [translation of Mathematics and modeling (Russian), 45-57, Akad. Nauk SSSR, Nauchn. Tsentr Biol. Issled., Pushchino, 1990; MR1089436 (92b:58189)], Selected translations, Selecta Math. 13 (1994), no. 1, 1-15. MR.1282313

[10] Faina S. Berezovskaya, Artem S. Novozhilov, and Georgy P. Karev, Population models with singular equilbrium, Math. Biosci. 208 (2007), no. 1, 270-299, DOI 10.1016/j.mbs.2006.10.006. MR.2330944 (2008j:92028)

[11] Georgy P. Karev, Artem S. Novozhilov, and Faina S. Berezovskaya, On the asymptotic behaviour of the solutions to the replicator equation, Math. Med. Biol. 28 (2011), no. 2, 89-110, DOI 10.1093/imammb/dqq006. MR2813300(2012h:91042)

Department of Mathematics, Howard University, Washington, DC 20059

E-mail address: fberezovskaya@howard.edu 